\title{
Las mejores estrategias que incrementan el uso de inmunizaciones y el rastreo de cáncer
}

Interventions That Increase Use of Adult Immunization and Cancer Screening Services: A Meta-Analysis. Stone E, Morton S Hulscher M, et al. Ann Intern Med. 2002;136:641-651.

\section{Objetivo}

Conocer la efectividad de distintas medidas (recordatorios, retroalimentación al proveedor, educación, incentivos financieros, acciones legislativas y regulatorias, cambios de la organización, campañas masivas en los medios) para promover inmunizaciones y rastreo de cáncer.

\section{Fuente de datos}

Medline, the Cochrane effective practice and Organization of Care Review Group register, revisiones sistemáticas previas y en la base de datos del Medicare Health Care Quality Improvement Project.

\section{Selección de estudios y extracción de los datos}

Inmunizaciones: estudios para vacunación contra la gripe y el neumococo.

Rastreo: estudios sobre mamografía, citología cervical (PAP) y sangre oculta en materia fecal (SOMF).

No se incluyeron trabajos sobre colonoscopía, ni sobre envíos postales o electrónicos masivos, ni 25 estudios que evaluaron distintas formas de una misma intervención o comparaciones idiosincráticas.De 552 artículos, 108 reunieron los criterios de inclusión. De los 108, 95 fueron ensayos clínicos aleatorizados y controlados y 13 incluyeron grupo control. Se evaluó el efecto de las distintas intervenciones utilizando el modelo de meta-regresión*

\section{Resultados Principales}

Efectividad de las intervenciones para mejorar el uso de prácticas preventivas.

\begin{tabular}{|c|c|c|c|c|}
\hline INTE & \multicolumn{4}{|c|}{ ODDS RATIO AJUSTADO (IC95\%) PARA CADA PRÁCTICA PREVENTIVA } \\
\hline & Inmunizaciones & Mamografía & PAP & SOMF \\
\hline $\begin{array}{l}\text { Cambios en la } \\
\text { organización }\end{array}$ & $16(11,2-22,8)$ & $2,47(1,78-4,24)$ & $3,03(2,56-3,58)$ & $17,6(12,3-25,2)$ \\
\hline \multicolumn{5}{|l|}{ Sobre pacientes } \\
\hline Incentivo económico & $3,42(2,89-4,06)$ & $2,74(1,78-4,24)$ & $2,82(2,35-3,38)$ & $1,82(1,35-2,46)$ \\
\hline Recordatorios & $2,52(2,24-2,82)$ & $2,31(1,97-2,70)$ & $1,74(1,58-1,92)$ & $2,75(1,90-3,97)$ \\
\hline Educación & $1,29(1,14-1,45)$ & $1,31(1,12-1,52)$ & $1,53(1,30-1,81)$ & $1,38(0,84-2,25)$ \\
\hline \multicolumn{5}{|c|}{ Sobre proveedores de la salud } \\
\hline Retroalimentación & $1,23(0,96-1,58)$ & $1,76(1,44-2,15)$ & $1,10(0,93-1,31)$ & $1,18(0,98-1,43)$ \\
\hline Recordatorio & $3,80(3,31-4,37)$ & $1,63(1,39-1,92)$ & $1,37(1,25-1,51)$ & $1,46(1,15-1,85)$ \\
\hline Educación & $3,21(2,24-4,61)$ & $1,99(1,58-2,51)$ & $1,72(1,39-2,13)$ & $3,01(1,98-4,56)$ \\
\hline Incentivo económico & $1,26(0,83-1,90)$ & & & \\
\hline
\end{tabular}

Para el análisis exploratorio de las características instrumentales de las intervenciones se tomó en cuenta las siguientes variables: 1) influencia social, 2) marketing, 3) uso de intervenciones con materiales visuales de impacto, 4) equipos de trabajo, 5) diseños basados en necesidades, barreras, incentivos, evaluaciones o teorías $6)$ nivel de apoyo del cuerpo directivo, 7) estrategias de aprendizaje activas.De éstas características salientes las intervenciones diseñadas sobre la base de necesidades o teoría (OR ajustado desde 1,44 a 2,16), las desarrolladas en equipos de trabajo (OR ajustado desde 1,22 a 17,9) y en las que se utilizaron materiales visuales de impacto (OR ajustado desde 1,19 a 3,25) fueron asociadas con un incremento del efecto estadísticamente significativo sobre tres o más prácticas preventivas.

\section{Conclusiones}

Las mejores estrategias son los cambios específicos en la organización. El trabajo en equipo (por problemas) y el diseño basado en el conocimiento de las necesidades y barreras son características que mejoran la efectividad de la intervención. Los recordatorios a los pacientes pueden adicionarse a las medidas anteriores, pudiendo ser considerados el estímulo financiero y la educación a los pacientes.

Fuente de financiamiento: Centers for Medicare \& Medicaid Services.

\section{que apoyan sus conclusiones.}

Los resultados de este estudio aportan información valiosa para decidir una intervención como parte de una estrategia, ya que algunas medidas ampliamente utilizadas durante años en las instituciones de salud demostraron ser poco efectivas.

Una de las principales limitaciones de este meta-análisis y que los mismos autores señalan, es la falta de información sobre costoefectividad* $^{*}$ en los artículos revisados. Es decir, sabiendo que la intervención es efectiva, conocer si el beneficio esperado, justifica el costo incremental ${ }^{*}$ por la implementación de dicha intervención. Conocer los beneficios, riesgos y costos de una intervención es fundamental para la toma de decisiones y sería de interés este análisis en futuros estudios.

Conclusiones del comentador: Los resultados del meta-análisis que muestran las mejores intervenciones para mejorar prácticas preventivas son muy sólidas ya que en el análisis utilizaron técnicas para ajustar las diferentes intervenciones entre sí. Sin embargo para evaluar la mejor intervención deberían tenerse en cuenta datos sobre costo-efectividad.

*Ver glosar io.

Dra. Cecilia B. Calvo [ Unidad de Medicina Familiar y Preventiva. Hospital Italiano de Buenos Aires ]

Referencias:

1.Briss PA, Rodewald LE, Hincan AR, et al.Reviews of evidence regarding interventions to improve vaccination coverage in children, adolescents and adults. The Task Force on Community Preventive Services.Am J Prev Med 2000:18:97-140.

2.Robson J, Boomla K, Fitzpatrick S, et al.Using nurses for preventive activities with computer assisted follow up:a randomized controlled trial.BMJ.1989;298:433-6. 